\title{
A Robust Emulation of Mechanical Loads Using a Disturbance-Observer
}

\author{
Kooksun Lee ${ }^{1}$, Jeongju Lee ${ }^{1}$, Juhoon Back ${ }^{2}$ D and Young Il Lee ${ }^{1, * \mathbb{D}}$ \\ 1 Department of Electrical and Information Engineering, Seoul National University of Science and Technology, \\ 232 Gongneung-ro, Nowon-gu, Seoul 01811, Korea; ksl1222@gmail.com (K.L.); joo0106@seoultech.ac.kr (J.L.) \\ 2 School of robotics, Kwangwoon University, 20 Kwangwoon-ro, Nowon-gu, Seoul 01897, Korea; \\ backhoon@kw.ac.kr \\ * Correspondence: yilee@seoultech.ac.kr; Tel.:+82-2-970-6544
}

Received: 11 May 2019; Accepted: 8 June 2019; Published: 12 June 2019

check for updates

\begin{abstract}
This paper deals with a new control strategy for the programmable dynamometer to emulate dynamic loads. The main idea is to employ the disturbance-observer-based design and take the nominal model involved in the disturbance-observer design as the desired dynamics to be emulated. Compared to previous approaches, the proposed approach does not require exact system parameters of the motor under test, and the range of emulation parameters is wider than previous results. A rigorous stability analysis, as well as a constructive design incorporating system uncertainty and the steady state error bound are presented. An experimental system is developed to verify the performance of the proposed method, and it is demonstrated that up to 20-times of inertia emulation with relatively small emulation error (speed error less than 6\%) is achieved and that various loads such as friction can be emulated.
\end{abstract}

Keywords: dynamic load emulation; disturbance-observer; performance; PMSM; dynamometer

\section{Introduction}

The characteristics of the motor itself are mainly tested by connecting mechanical components, designed for mass or friction load, to the motor shaft [1]. On the other hand, the test should be performed under various load conditions if the motor is developed for high reliability applications such as electric vehicles [2,3], wind power generators [4], aircraft [5], etc. In this case, it is customary to test the motor and its driving circuits together by connecting them to another electric machine, usually called a programmable dynamometer, which produces mechanical torque that is supposed to act on the motor as load torque in the real operation.

In recent research, several methods for the dynamic load emulation were proposed, in which the torque of the programmable dynamometer was determined by some closed-loop algorithms so that the dynamics of the emulator became that of a desired one. One of early results given in [6] employed the inverse model of the system. The structure proposed in [6] was simple and easy to understand, so that it is convenient to implement in various applications. In [4], the authors developed an algorithm to emulate the effect of an inertia using a hardware-in-the-loop wind turbine simulator. Nonlinear and possibly discontinuous load torques were emulated in [7]. The performance of these approaches was limited. The approach in [6] was sensitive to measurement noises, since it involved the inverse of a strictly proper transfer function, and it is not possible to emulate an inertia that is much larger than the actual inertia of the test-rig [8]. In [8], the authors proposed an alternative controller, called feedforward-tracking, where a feedforward term was added to compensate the motor torque, and the load emulation was performed by an additional speed control loop. This controller has been well accepted in the industry, and its performance has been verified through various experimental 
results, e.g., emulation of a flexible-shaft [9], emulation of the model by applying the parametric system identification method [5], and some experiments using industrial drives [10]. It is noted that in the above approaches, the effect of plant uncertainties on system stability and performance were not explicitly addressed.

In this paper, a disturbance-observer (DOB)-based emulation scheme is presented. A preliminary result has been reported in [11], where the basic idea was introduced. DOB is a robust output feedback controller and has been successfully employed to compensate the external disturbances and plant uncertainties in many real applications such as robust servo control [12] and robust track-following of an optical disk drive [13]. See the survey [14] for various DOB-based controller and related methods. Roughly speaking, the dynamic inverse of the nominal model of the plant is used to estimate the input combined with the disturbance, and the disturbance is estimated by subtracting the input from the proceeding signal. In fact, the resulting estimate of disturbance is different from the actual external disturbance due to plant uncertainty, and thus, it is the combined effect of plant uncertainty and the external disturbance. One of the key benefits of $\mathrm{DOB}$ is that it can recover the performance of a nominal closed-loop system that is composed of the nominal plant and the controller designed for the nominal system $[15,16]$. We call this property nominal performance recovery.

The nominal performance recovery property of DOB is exploited to obtain a robust emulation scheme, and the key idea is to regard the dynamics to be emulated (e.g., a mechanical system with a desired inertia and damping) as the nominal plant in the DOB scheme. This new idea enables us to successfully address the emulation problem considering the plant uncertainty. We emphasize that this robustness against plant uncertainty in the emulation problem makes our result different from previous emulation approaches mentioned above. Another important contribution is that the range of emulation parameters is wider than previous results, and a rigorous stability analysis in the state space is carried out, which was not taken into account in previous research. Note that this time-domain analysis helps us to consider the nonlinear load emulation. Finally, the efficacy of the proposed method is also verified through various experiments for both linear and nonlinear loads.

The rest of this paper is organized as follows. Section 2 formulates the problem in this paper. Section 3 presents the main idea of the proposed method to emulate the mechanical load and the load torque. In this section, the performance of the proposed method is guaranteed by a rigorous stability analysis. In Section 4, the proposed method is verified by some experiments using two electrical machines. Finally, some concluding remarks are shown in Section 5.

\section{Problem Formulation}

We consider a motor test system, shown in Figure 1, which is composed of an electric motor to be tested and another electric machine, called a programmable dynamometer.

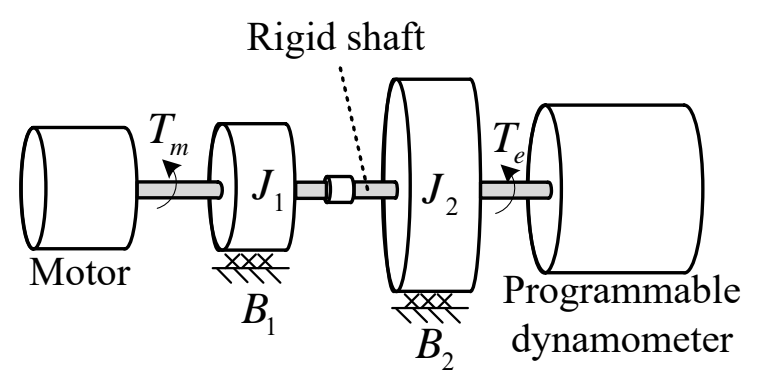

Figure 1. Emulation of mechanical loads using a dynamometer.

Two electrical machines in the system are connected by a rigid shaft, and thus, the dynamics is given by:

$$
J \dot{\omega}_{m}+B \omega_{m}=T_{m}+T_{e}+d
$$


where $J=J_{1}+J_{2}$ is the lumped inertia of the shaft, which includes the inertia of the driving motor and that of the load machine, $B=B_{1}+B_{2}$ is the friction coefficient, which is also a lumped parameter, $T_{m}$ is the electrical torque generated by the motor under test, $T_{e}$ is the torque from the dynamometer, $\omega_{m}$ is the rotating speed of the shaft, and $d$ is the disturbance torque.

Suppose that the motor under test is connected to a desired mechanical load with inertia $J_{e m}$ and friction $B_{e m}$. In this case, the dynamics becomes:

$$
J_{e m} \dot{\omega}_{e m}+B_{e m} \omega_{e m}=T_{m}-T_{l}
$$

where $\omega_{e m}$ and $T_{l}$ are the rotating speed and the load torque of the desired dynamics to emulate. The control objective is to manipulate $T_{e}$ so that $\omega_{m}$ of (1) follows $\omega_{e m}$ of (2) as close as possible. The transfer functions of (1) and (2) are derived as follows:

$$
\begin{aligned}
\omega_{m}(s) & =G(s)\left(T_{m}(s)+T_{e}(s)+d(s)\right), \\
\omega_{e m}(s) & =G_{e m}(s)\left(T_{m}(s)-T_{l}(s)\right)
\end{aligned}
$$

where:

$$
\begin{aligned}
G(s) & =\frac{1}{J s+B^{\prime}} \\
G_{e m}(s) & =\frac{1}{J_{e m} s+B_{e m}} .
\end{aligned}
$$

In many previous research works, the controllers were designed in the frequency-domain considering the transfer functions (4). In [6], the dynamometer torque was generated as $T_{e}(s)=$ $\left(B-B_{e m}\right) \omega_{m}(s)+\left(J-J_{e m}\right) s \omega_{m}(s)$ in order to correct the speed response $\omega_{m}(s) / T_{m}(s)$ of $(4 \mathrm{a})$ into (4b), and [8] proposed to use $T_{e}(s)=-T_{m}(s)+G_{t}(s)\left(G_{c o m p}(s) G_{e m}(s) T_{m}(s)-\omega_{m}(s)\right)$ where $G_{c o m p}(s)=\frac{1+G_{t}(s) G(s)}{G_{t}(s) G(s)}$ with a design parameter $G_{t}(s)$. Both $[6,8]$ neglected the disturbance torque and load torque and required the knowledge of the parameters $J$ and $B$, but the effects of the parameter uncertainties were not addressed in detail. Note that the performance of the controller was degraded by the parameter uncertainties in many cases.

Unlike the earlier works, the disturbance/load torque will be taken into account, and it is assumed that the exact values of the parameter $J$ and $B$ are unknown. Instead, the following assumptions were made.

Assumption 1. It is assumed that the plant parameters J and B are uncertain, but belong to the following bounded sets:

$$
\begin{aligned}
& \mathcal{J}=\{J \mid 0<\underline{J} \leq J \leq \bar{J}\}, \\
& \mathcal{B}=\{B \mid 0<\underline{B} \leq B \leq \bar{B}\} .
\end{aligned}
$$

Assumption 2. The torques $T_{m}, T_{l}$ and the external disturbance d are twice differentiable and bounded, i.e., there exists $\left|T_{m}\right| \leq T_{m}^{+},\left|\dot{T}_{m}\right| \leq \dot{T}_{m}^{+},|\dot{d}| \leq \dot{d}^{+}$with known constants $T_{m}^{+}, \dot{T}_{m}^{+}$, and $\dot{d}^{+}$, respectively.

In the following sections, a new emulation method for generating the dynamometer torque $T_{e}$ will be proposed with its performance/stability analysis.

\section{Robust Load Emulation Controller: Theory}

Figure 2 illustrates the application of the conventional DOB to load emulation, which is similar to the servo-control scheme of [12]. Compared with [12], the figure has some distinctive features such that the load torque $T_{l}$ is added, and the nominal model of the plant is replaced by the emulating dynamics $G_{e m}(s)$. 


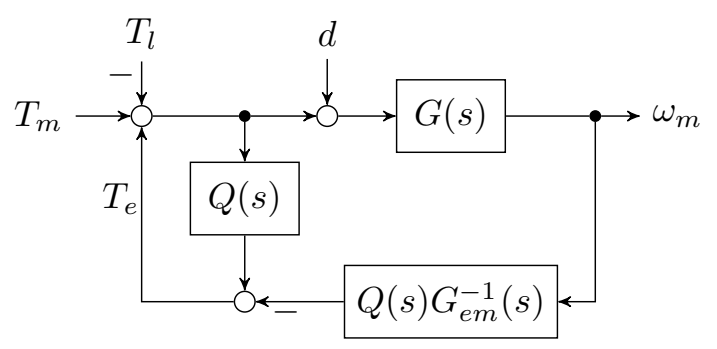

Figure 2. The concept of the proposed method using the disturbance-observer (DOB).

From this figure, the closed-loop system can be written as:

$$
\begin{aligned}
\omega_{m}(s) & =\mathcal{T}(s)\left\{T_{m}(s)-T_{l}(s)+(1-Q(s)) d(s)\right\}, \\
\mathcal{T}(s) & =\frac{G(s) G_{e m}(s)}{G_{e m}(s)+\left(G(s)-G_{e m}(s)\right) Q(s)}
\end{aligned}
$$

where $Q(s)$ is the low pass filter with unity DC gain $\left(\lim _{\omega \rightarrow 0} Q(j \omega)=1\right)$, called the Q-filter in the conventional DOB structure. It can be observed that the closed-loop system (5) will behave like the desired emulation dynamics ( $3 b$ ) in the low frequency range.

A state-space emulation method that can be expressed as follows will be developed:

$$
\begin{aligned}
T_{e} & =f_{e}\left(q, \omega_{m}, T_{m}, T_{l}\right), \\
\dot{q} & =f_{q}\left(q, \omega_{m}, T_{m}, T_{l}\right)
\end{aligned}
$$

where $q$ is the state of the controller and $f_{e}$ and $f_{q}$ are functions to be designed. Thus, it is required to redraw Figure 2 so that the $T_{e}$ can be made from $\omega_{m}, T_{m}$, and $T_{l}$ is shown clearly as per the following subsection.

\subsection{Disturbance Observer-Based Emulation Method}

From Figure 2, the dynamometer torque is generated by:

$$
T_{e}(s)=Q(s)\left(T_{m}(s)-T_{l}(s)+T_{e}(s)\right)-Q(s) G_{e m}^{-1}(s) \omega_{m}(s)
$$

where the Q-filters are chosen as $Q(s)=\frac{1}{\delta s+1}$ (This is a Type-I Q-filter in [12]. Since the proposed emulation method is based on DOB (Figure 2), the higher-order Q-filters are also available in order to improve the emulating performance.) with design parameters $\delta>0$ in this paper. Note that there is an algebraic loop of $T_{e}(s)$ in (7). Thus, it can be rewritten as:

$$
T_{e}(s)=\frac{1}{\delta s}\left(T_{m}(s)-T_{l}(s)-B_{e m} \omega_{m}(s)\right)-\frac{1}{\delta} J_{e m} \omega_{m}(s) .
$$

Let $q=\frac{1}{s}\left(T_{m}(s)-T_{l}(s)-B_{e m} \omega_{m}(s)\right)$, then a state-space realization of (8) is obtained as follows:

$$
\begin{aligned}
T_{e} & =\frac{1}{\delta}\left(q-J_{e m} \omega_{m}\right), \\
\dot{q} & =T_{m}-T_{l}-B_{e m} \omega_{m},
\end{aligned}
$$

which could be considered as the implementations of $f_{e}(6 \mathrm{a})$ and $f_{q}(6 \mathrm{~b})$. 
One can see that (9) can be drawn as Figure 3, and it is a particular implementation of Figure 2. It will be proven later that the performance of this structure is guaranteed as:

$$
\limsup _{t \rightarrow \infty}\left|\omega_{m}(t)-\omega_{e m}(t)\right| \leq \epsilon^{*}
$$

where the trajectory of the actual system (1) follows the desired emulation dynamics (2) within a bound $\epsilon^{*}$.

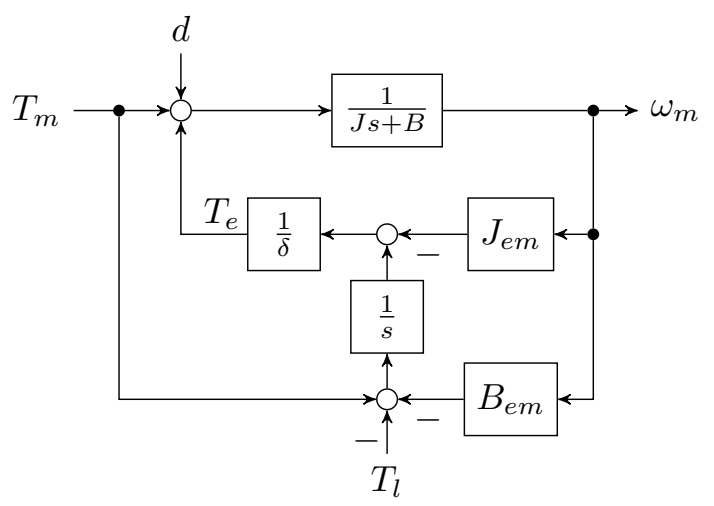

Figure 3. Proposed method to determine the torque of a dynamometer $\left(T_{e}\right)$ for the dynamic emulation of $(3 b)$.

Figure 4 shows the Bode diagram of the real dynamics (4a), desired dynamics (4b), and emulated dynamics (5) for different $\delta^{\prime}$ s. In the diagram, it is clear that the frequency response of the emulated dynamics is close to that of the desired dynamics in the low-frequency range and that this frequency range is increased as a smaller $\delta$ is used.

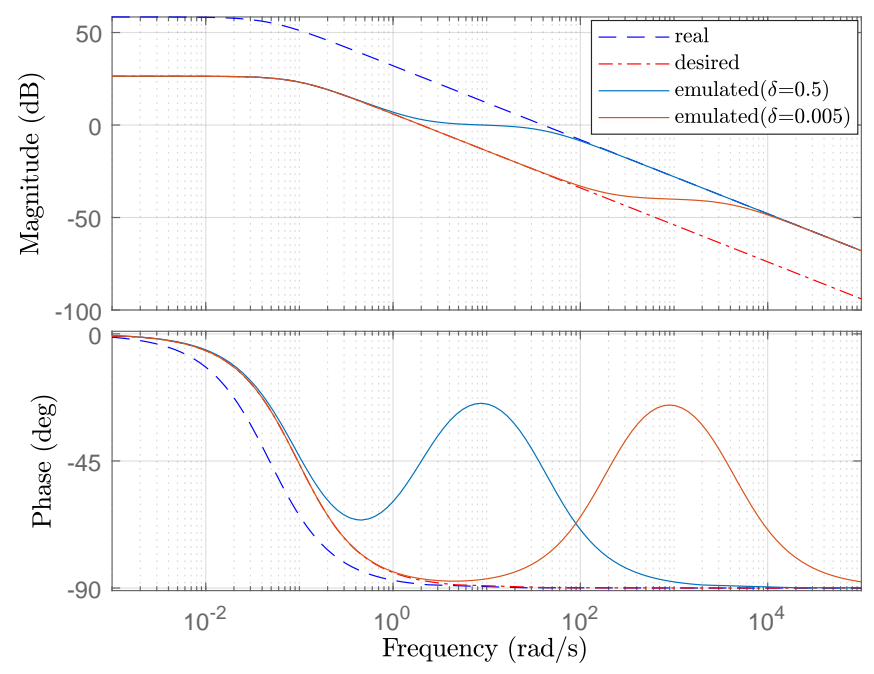

Figure 4. Frequency responses of the real dynamics (4a), desired dynamics (4b) and emulated dynamics (5) for different $\delta^{\prime}$ s with $J_{e m}=20 \mathrm{~J}$ and $B_{e m}=40 B$.

\subsection{Performance Analysis}

This section presents the emulating performance of the proposed method based on the singular perturbation theory [17].

Lemma 1. There exists a $\delta>0$ such that the closed-loop system (1) and (9) is exponentially stable. 
Proof. Let us denote $T_{e}$ as $\eta$ for convenience. It is a matter of simple algebra to show that the closed-loop system becomes:

$$
\begin{gathered}
\dot{\omega}_{m}=\frac{1}{J}\left(T_{m}-B \omega_{m}+\eta+d\right), \\
\delta \dot{\eta}=-\frac{J_{e m}}{J} \eta+\left(\frac{J_{e m}}{J} B-B_{e m}\right) \omega_{m}+\left(1-\frac{J_{e m}}{J}\right) T_{m}-T_{l}-\frac{J_{e m}}{J} d .
\end{gathered}
$$

This is the linear system (with respect to the inputs $T_{m}, T_{l}$, and $d$, respectively.) whose system matrix is given by:

$$
\left[\begin{array}{cc}
-\frac{B}{J} & \frac{1}{J} \\
\frac{1}{\delta}\left(\frac{J_{e m}}{J} B-B_{e m}\right) & -\frac{1}{\delta} \frac{J_{e m}}{J}
\end{array}\right],
$$

and this matrix is Hurwitz for some $\delta>0$. This completes the proof.

In Lemma 1, the closed-loop system (11a), (11b) is written in the standard singular perturbation form [17]. Based on the singular perturbation theory, the closed-loop system is divided by the slow variable $\left(\omega_{m}\right)$ and the fast variable $(\eta)$ with the time-separation parameter $\delta$. For sufficiently small $\delta \approx 0$, the slow variable is frozen, while the fast variable $\eta$ converges to its equilibrium, namely $\eta^{*}$ :

$$
\eta^{*}=\left(B-\frac{J}{J_{e m}} B_{e m}\right) \omega_{m}+\left(\frac{J}{J_{e m}}-1\right) T_{m}-\frac{J}{J_{e m}} T_{l}-d .
$$

Suppose that the fast variable has converged $\left(\eta=\eta^{*}\right)$; the slow variable behaves like the the desired emulation dynamics (2): $J_{e m} \dot{\omega}_{m}+B_{e m} \omega_{m}=T_{m}-T_{l}$ under consideration in this paper. This kind of approach was also shown in [11], but the stability analysis was limited by perfect time scale separation, in which the goal (2) was achieved by assuming arbitrary small $\delta \approx 0$.

Remark 1. In recent DOB approaches [15,16], the standard singular perturbation form was obtained by some coordinate transforms. On the other hand, no additional procedure is required in the proposed method due to the simple structure (9).

Now, the realistic condition $(\delta>0)$ is considered in this paper. To evaluate the emulating performance, the emulating error $e:=\omega_{m}-\omega_{e m}$ is taken into account with the the fast variable by the coordinate change $\tilde{\eta}:=\eta-\eta^{*}$,

$$
\begin{aligned}
\dot{e} & =-\frac{B_{e m}}{J_{e m}} e+\frac{1}{J} \tilde{\eta}, \\
\delta \dot{\tilde{\eta}} & =-\frac{J_{e m}}{J} \tilde{\eta}-\delta \dot{\eta}^{*} .
\end{aligned}
$$

In (14b), $\dot{\eta}^{*}$ is directly computed from (13) as follows:

$$
\dot{\eta}^{*}=\gamma_{1} \theta-\gamma_{1} \frac{J}{J_{e m}} B_{e m} e-u(t)
$$

where $u(t)=-\gamma_{1} J \dot{\omega}_{e m}+\gamma_{2} \dot{T}_{m}+\frac{J}{J_{e m}} \dot{T}_{l}+\dot{d} ., \gamma_{1}=\frac{B}{J}-\frac{B_{e m}}{J_{e m}}$, and $\gamma_{2}=1-\frac{J}{J_{e m}}$, respectively. It can be easily shown that the response of the emulating error is determined by the stable transfer function:

$$
\frac{e(s)}{u(s)}=\frac{\frac{1}{J}}{s^{2}+\left(\frac{1}{\delta} \frac{J_{e m}}{J}+\frac{B}{J}\right) s+\frac{1}{\delta} \frac{B_{e m}}{J}}
$$

which is computed from (14) and (15). In (16), the input signal $u(t)$ is bounded (see Lemma 2 for more detail) under the assumptions in this paper; it follows that there exists a positive constant $\epsilon$, the emulating error is bounded by $|e(t)|<\epsilon, \forall t$. 
Lemma 2. Under Assumptions 1-2, the conservative bound of the input signal $\bar{u}>u(t), \forall t$ can be computed by:

$$
\bar{u}=\bar{\gamma}_{1} \bar{J} \dot{\omega}_{e m}^{+}+\bar{\gamma}_{2} \dot{T}_{m}^{+}+\frac{\bar{J}}{J_{e m}} \dot{T}_{l}^{+}+\dot{d}^{+}
$$

where the bound $\dot{\omega}_{e m}^{+} \geq\left|\dot{\omega}_{e m}\right|$ is obtained using the knowledge of the desired emulation dynamics (2), and the constants are computed by:

$$
\begin{aligned}
& \bar{\gamma}_{1}=\max \left\{\left|\frac{\underline{B}}{\bar{J}}-\frac{B_{e m}}{J_{e m}}\right|,\left|\frac{\bar{B}}{\underline{J}}-\frac{B_{e m}}{J_{e m}}\right|\right\}, \\
& \bar{\gamma}_{2}=\max \left\{\left|1-\frac{J}{J_{e m}}\right|,\left|1-\frac{\bar{J}}{J_{e m}}\right|\right\} .
\end{aligned}
$$

The proof is omitted, since it is obvious.

Finally, the emulating performance of the proposed method is guaranteed by the time-domain analysis, in which the Lyapunov stability analysis of the proposed method is shown by the following theorem.

Theorem 1. For the given desired bound $\epsilon^{*}>0$, there exists a constant $\delta^{*}>0$ such that for any $0<\delta<\delta^{*}$, the trajectory $\omega_{m}(t)$ of the closed-loop system (14) and (15) satisfies (10).

Proof. The dynamics $(e, \tilde{\eta})$ is compactly rewritten by:

$$
\frac{d}{d t}\left[\begin{array}{l}
e \\
\tilde{\eta}
\end{array}\right]=\left[\begin{array}{cc}
-\frac{B_{e m}}{J_{e m}} & \frac{1}{J} \\
\frac{J B_{e m}}{J_{e m}} \gamma_{1} & -\frac{1}{\delta} \frac{J_{e m}}{J}-\gamma_{1}
\end{array}\right]\left[\begin{array}{l}
e \\
\tilde{\eta}
\end{array}\right]+\left[\begin{array}{l}
0 \\
1
\end{array}\right] u(t)
$$

Consider a Lyapunov function candidate:

$$
V=\frac{1}{2} \rho_{1} e^{2}+\frac{1}{2} \rho_{2} \tilde{\eta}^{2}>0
$$

with the positive constants $\rho_{1}$ and $\rho_{2}$ to be determined. Then,

$$
\begin{aligned}
\dot{V} & =-\rho_{1} \frac{B_{e m}}{J_{e m}} e^{2}-\rho_{2}\left(\frac{1}{\delta} \frac{J_{e m}}{J}+\gamma_{1}\right) \theta^{2}+\left(\rho_{1} \frac{1}{J}+\rho_{2} \frac{J}{J_{e m}} B_{e m} \gamma_{1}\right) e \tilde{\eta}+\rho_{2} \tilde{\eta} u(t) \\
& \leq\left(\frac{1}{2}-\frac{\rho_{1} B_{e m}}{J_{e m}}\right) e^{2}-\frac{\rho_{2} J_{e m}}{\delta J} \tilde{\eta}^{2}+M(\mu) \tilde{\eta}^{2}+\mu \bar{u}^{2}
\end{aligned}
$$

where:

$$
M(\mu)=-\rho_{2} \gamma_{1}+\frac{1}{2}\left(\rho_{1} \frac{1}{J}+\rho_{2} \frac{J}{J_{e m}} B_{e m} \gamma_{1}\right)^{2}+\frac{1}{4 \mu} \rho_{2}^{2}
$$

and $\mu$ is a positive constant from the Young's inequality.

From (18), the global exponential stability of the origin of the unforced system will be investigated with $\bar{u}=0$. By choosing $\rho_{1}>\frac{J_{e m}}{B_{e m}}, \rho_{2}>0$, and $\delta^{*}$ such that:

$$
-\frac{1}{\delta^{*}} \frac{\rho_{2} J_{e m}}{J}+M(\mu) \leq-\frac{1}{2}
$$


this guarantees the exponential stability of the origin for any $\delta<\delta^{*}$. Now, the input $\bar{u} \neq 0$ is allowed to obtain $\dot{V} \leq-k V+\mu \bar{u}^{2}$ where $k=\frac{1}{\max \left\{\rho_{1}, \rho_{2}\right\}}$. By the comparison lemma, we have:

$$
\limsup _{t \rightarrow \infty} V(t)=\frac{1}{2} \limsup _{t \rightarrow \infty}\left(\rho_{1} e^{2}+\rho_{2} \tilde{\eta}^{2}\right) \leq \frac{\mu}{k} \bar{u}^{2}
$$

from which it follows that:

$$
\limsup _{t \rightarrow \infty}|e(t)| \leq \sqrt{\frac{2}{\rho_{1}} \frac{\mu}{k}} \bar{u} .
$$

Since $\mu$ is an arbitrary constant, (20) completes the proof. In other words, for a given $\epsilon>0$, there exists $\delta^{*}$ such that for any $\delta$ satisfying $0<\delta<\delta^{*}$, it holds that:

$$
\limsup _{t \rightarrow \infty}|e(t)|=\limsup _{t \rightarrow \infty}\left|\omega_{m}(t)-\omega_{e m}(t)\right| \leq \epsilon^{*} .
$$

The analysis of this paper implies that sufficiently small $\delta<\delta^{*}$ is required to guarantee the desired performance lim sup $|e(t)| \leq \epsilon^{*}$. One can obtain $\delta^{*}$ by computing the conservative bounds of the signals or via repeated experiments, as shown in the next section.

\section{Experimental Result}

In this section, experimental results are provided to show that: (1) the proposed method emulates a wide range of the linear dynamic loads, and it also compensates the effects of the disturbance torque $d$; (2) the emulation of the desired dynamics can be performed in the presence of various load torques $T_{l}$.

Figures 5 and 6 show the experimental setup and its block diagram, respectively, to evaluate the performance of the proposed method (Figure 3). The electrical parameters of the PMSM and the induction motor are described in Table 1 . The proposed method was implemented in a microprocessor, TMS320f28377D with a 200-MHz clock frequency. Three-phase currents $\left(i_{a}, i_{b}, i_{c}\right)$ were measured by the current sensors, while three-phase voltages $\left(v_{a}, v_{b}, v_{c}\right)$ were applied to the PMSM through the switching devices with the switching frequency $f_{s}=10 \mathrm{kHz}$ and the DC-link voltage $V_{d c}=100 \mathrm{~V}$. The PI-gains of the dq-axis current controllers were chosen as $K_{p c}=L_{q} \omega_{c c}$ (or $L_{d} \omega_{c c}$ ) and $K_{i c}=R_{s} \omega_{c c}$ where $\omega_{c c}=2 \pi f_{s} / 10$ denotes the desired bandwidth of the current controllers [18]. The rotating speed of the shaft was computed by the numerical differentiation: $\omega_{m}=\frac{d \theta_{m}}{d t}$ where the shaft angle $\theta_{m}$ was measured by a $\sin / \cos$ encoder in order to alleviate some problems related to the numerical differentiation, since this provided significantly higher resolution of the measurement than conventional optical encoders. All measurements were monitored by a laptop every $0.3 \mathrm{~ms}$ using the CAN protocol.

\subsection{Implementation of the Proposed Method with the Electrical Machine}

In the motor side of Figure 6, the induction motor was used to generate $T_{m}$ with a commercial motor drive (Varispeed G7, YASKAWA), where the reference of $T_{m}$ is denoted by $T_{m}^{*}$. Various operations were carried out using the commercial motor drive to test the performance of the load emulator. In the dynamometer side, the PMSM was used to generate $T_{e}$ following the output of the proposed disturbance-observer $T_{e}^{*}$. The emulation was performed by a cascade control scheme, i.e., the proposed disturbance-observer (Figure 3) generated $T_{e}^{*}$ in the outer-loop, and the conventional field-oriented current control formed the inner-loop. In all experiments, the rotating speed and the torques were measured, and the results were compared with the response of the desired dynamics obtained by the numerical simulation. The measurement $\omega_{m}(t)$ followed the simulation result $\omega_{e m}(t)$, if the emulation was successfully performed by the proposed method. 


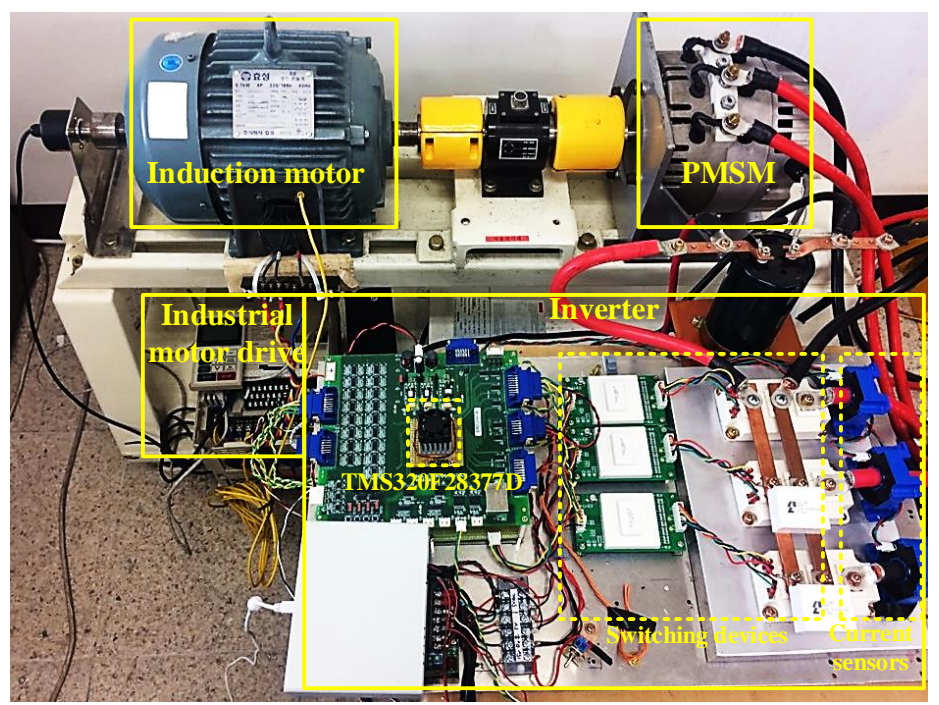

Figure 5. Experimental system.

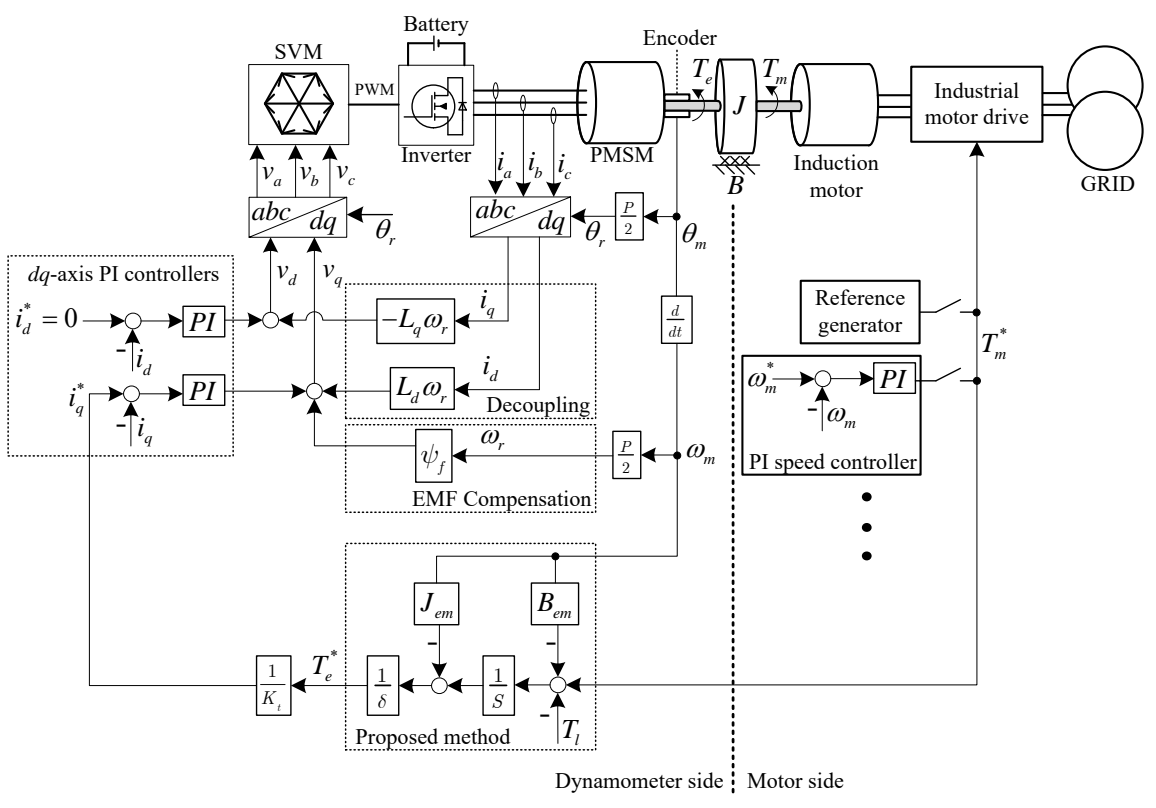

Figure 6. Block diagram of the experimental system.

Table 1. Electrical parameters of the PMSM and the induction motor.

\begin{tabular}{cc}
\hline \multicolumn{2}{c}{ PMSM } \\
\hline Number of poles $(P)$ & 8 \\
Rated torque $\left(T_{e, \text { rated }}\right)$ & $22.5 \mathrm{Nm}$ \\
Rated speed $\left(\omega_{m, \text { rated }}\right)$ & $3000 \mathrm{RPM}$ \\
Rotor flux linkage $\left(\psi_{f}\right)$ & $0.025 \mathrm{~Wb}$ \\
Stator inductance $\left(L_{d}\right.$ and $\left.L_{q}\right)$ & $0.12 \mathrm{mH}$ \\
Stator resistance $\left(R_{s}\right)$ & $9.5 \mathrm{~m} \Omega$ \\
\hline Induction Motor & \\
\hline Number of poles & 8 \\
Rated torque & $20 \mathrm{Nm}$ \\
Rated speed & $1730 \mathrm{RPM}$ \\
Slip ratio & $3.8 \%$ \\
\hline
\end{tabular}


In Figure 6, the proposed method was applied under the following assumptions:

1. The tracking dynamics of the $d q$-axis current controllers and the torque controller in the industrial motor drive were negligible due to their fast responses; thus, it was assumed that $i_{d}^{*}=i_{d}, i_{q}^{*}=i_{q}$, and $T_{m}^{*}=T_{m}$, respectively.

2. Since $i_{d}=0$ was controlled by the $d$-axis controller, the torque was developed by the relation $T_{e}=K_{t} i_{q}$ where $K_{t}=\frac{3}{2} \frac{P}{2} \psi_{f}$ is the well-known torque constant.

These kinds of assumptions have been adopted by many previous researchers.

\subsection{Experimental Results: Linear Loads}

Figure 7 shows the responses when the sinusoidal reference $T_{m}=\sin (2 \pi t) \mathrm{Nm}$ was applied to the motor. In the experiments, the desired dynamic loads were chosen as $J_{e m}=J=0.025 \mathrm{~kg} \cdot \mathrm{m}^{2}$, $B_{e m}=B=0.0012 \mathrm{Nms} / \mathrm{rad}$, and $T_{l}=0$, respectively. The design parameter $\delta$ of the controller was changed to demonstrate the performance. In Figure 7a, it can be observed that the emulating error was decreased as $\delta$ became smaller, while Figure $7 \mathrm{~b}$ shows that the disturbance torque $d$ was compensated by the dynamometer. Since (13) holds $T_{e}=K_{t} i_{q}=-d$ in this case, one can choose the value of $\delta$ by similar procedures as shown in Figure 7. For the rest of experiments, we fixed $\delta=0.01$. This value was chosen to compromise between the tracking accuracy and the smoothness of $T_{e}$. Note that in the case with $\delta=0.005$, we had more accurate tracking error (Figure $7 \mathrm{~b}$ ), while in the case with $\delta=0.05$, we had a smoother profile in $T_{e}$.

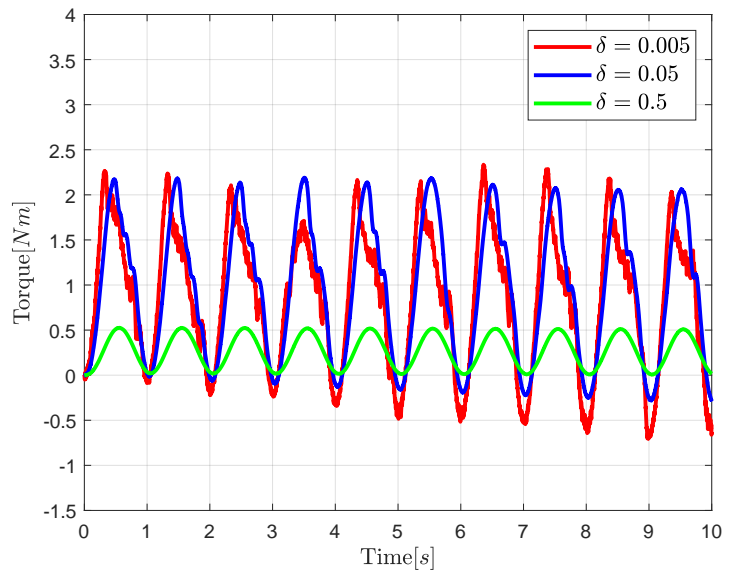

(a)

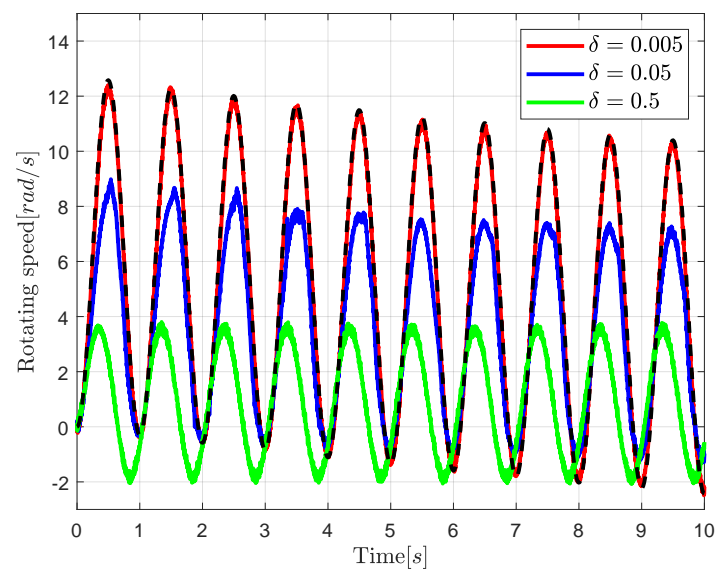

(b)

Figure 7. The responses with various design parameters $\delta: T_{m}=\sin (2 \pi t) \mathrm{Nm}$. (a) The measurement of the dynamometer torque $T_{e}$. (b) The measurement of the rotating speed $\omega_{m}$ (solid), and the desired one $\omega_{e m}$ (dashed) obtained by numerical simulation.

Remark 2. In fact, the disturbance torque dis unknown in practice. The proposed method compensates the total disturbance (13) including the estimation of the disturbance, as discussed in earlier.

Figure 8 shows the responses with respect to various dynamic loads $J_{e m}$ to emulate. The step input $T_{m}=5 u(t) \mathrm{Nm}$ was applied to the motor, where $u(t)$ denotes the unit step input $\left(u\left(t-t_{0}\right)=1\right.$ if $t>t_{0}$, otherwise it is zero). At $60 \mathrm{~s}$, the load torque $T_{l}=10 u(t-60) \mathrm{Nm}$ to emulate was also applied. In the simulation, the emulation error $\left(\omega_{e m}(t)-\omega_{m}(t)\right) / \omega_{e m, s s}$ was less than $6 \%$ when the response of the desired emulation dynamics (2) was relatively fast with small $J_{e m}=J$. The emulation error became smaller in the case of larger $J_{e m}>J$. 


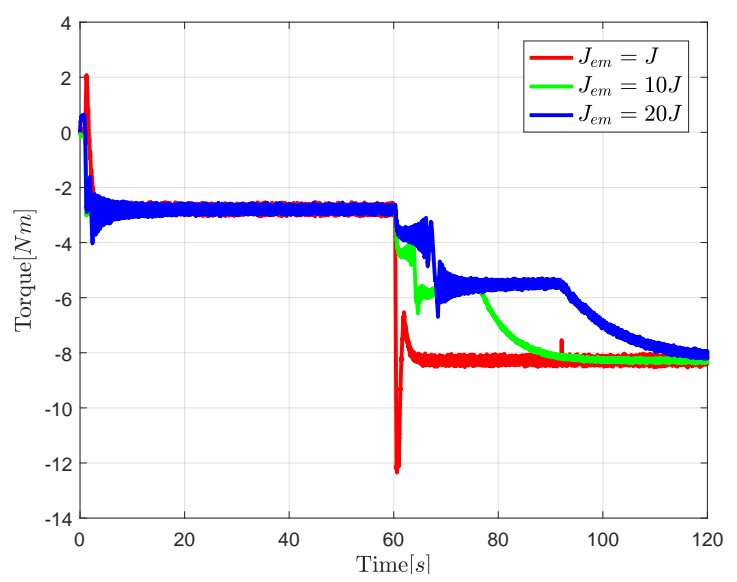

(a)

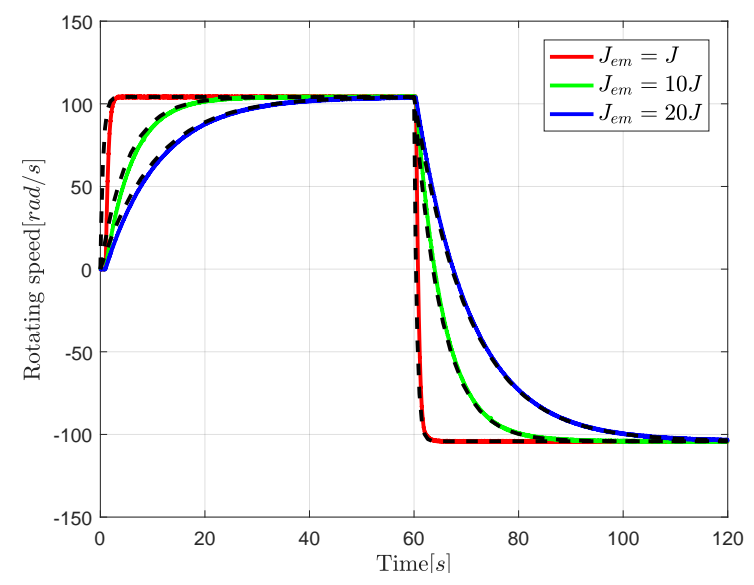

(b)

Figure 8. The responses with various dynamic loads to emulate $\left(B_{e m}=40 \mathrm{~B}\right.$ is fixed): $T_{m}=5 u(t) \mathrm{Nm}$ and $T_{L}=10 u(t-60) \mathrm{Nm}$. (a) The measurement of the dynamometer torque $T_{e}$. (b) The measurement of the rotating speed $\omega_{m}$ (solid), and the desired one $\omega_{e m}$ (dashed) obtained by numerical simulation.

Remark 3. In many previous research works, the experiments were performed under relatively limited conditions compared to the results in this paper, e.g., $J_{e m}=10 \mathrm{~J}$ for the linear load $[8,9], J_{e m}=2 \mathrm{~J}$ for the nonlinear loads [19], and $J_{e m}=3 \mathrm{~J}$ with the industrial motor drives (SIEMENS) using HIL devices [10], respectively.

Figure 9 shows the case when the motor side was driven by a conventional PI-type speed controller:

$$
T_{m}(s)=\frac{K_{p s} s+K_{i s}}{s}\left(\omega_{m}^{*}(s)-\omega_{m}(s)\right)
$$

where $K_{p s}$ and $K_{i s}$ are the PI gains of the controller; the reference speed and the load torque are given by $\omega_{m}^{*}=100 u(t) \mathrm{rad} / \mathrm{s}$ and $T_{l}=10 u(t-60) \mathrm{Nm}$ in this experiment. The desired dynamic loads were chosen as $J_{e m}=20 \mathrm{~J}$ and $B_{e m}=40 \mathrm{~B}$. Note that the motor torque was developed by the closed-loop controller (21), unlike previous experiments; thus, the simulation results to be compared were also given by closed-loop system:

$$
\begin{aligned}
T_{e m}(s) & =\frac{K_{p s} s+K_{i s}}{s}\left(\omega_{m}^{*}(s)-\omega_{e m}(s)\right), \\
\omega_{e m}(s) & =G_{e m}(s)\left(T_{e m}(s)-T_{l}(s)\right),
\end{aligned}
$$

where $T_{e m}$ and $\omega_{e m}$ correspond to the measurement $T_{m}$ and $\omega_{m}$. In addition, the saturation function was added to limit the motor torque within $\left|T_{m}\right|<20 \mathrm{Nm}$. In the figure, it can be observed that both the torque and the rotating speed of the motor-side were emulated with very small errors ( $\omega_{m} \approx \omega_{e m}$ and $\left.T_{m} \approx T_{e m}\right)$. 

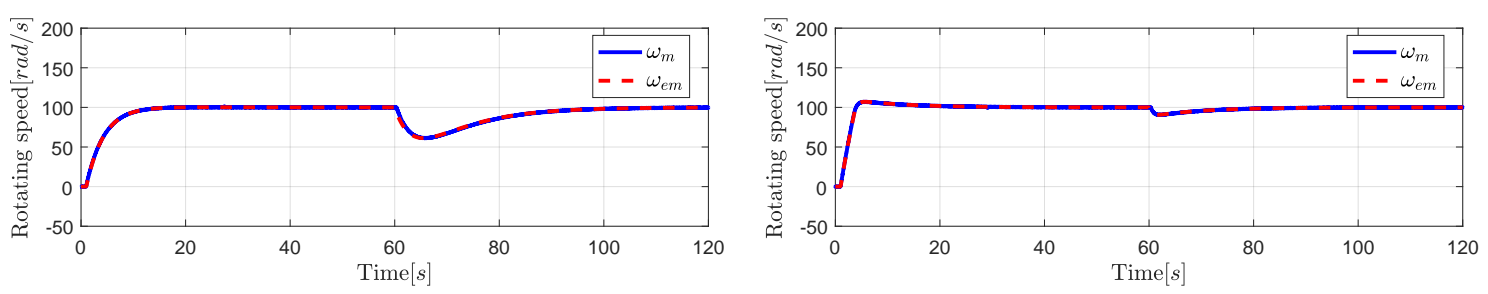

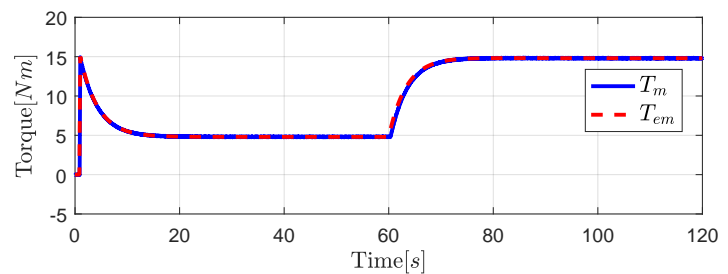

(a)

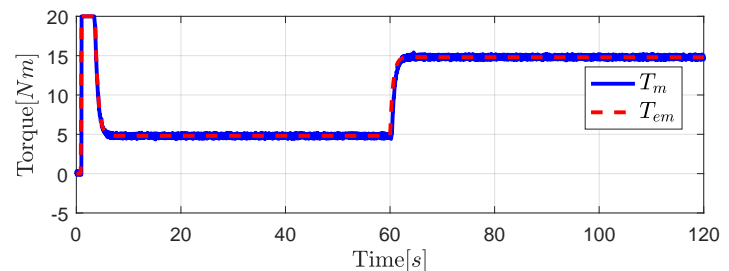

(b)

Figure 9. The responses of the motor torque $T_{m}$ with the conventional PI-type speed controller (21): $J_{e m}=20 \mathrm{~J}$ and $B_{e m}=40 \mathrm{~B}$. (a) The experimental results (solid) and the desired response (dashed), $K_{p s}=0.15$ and $K_{i s}=0.015$. (b) The experimental results (solid) and the desired response (dashed), $K_{p s}=0.9$ and $K_{i s}=0.09$.

\subsection{Experimental Results: Nonlinear Loads}

In this experiment, the performance of the proposed method was verified by emulating a nonlinear friction load:

$$
\begin{aligned}
J_{e m} \dot{\omega}_{e m} & =-B_{e m} \omega_{e m}+T_{m}+R_{r} F_{f} \\
m \dot{V}_{v} & =-F_{f}, \omega_{v}=\frac{V_{v}}{R_{r}} \\
F_{f} & =m g \mu(\lambda)
\end{aligned}
$$

where $m$ is the mass, $V_{v}$ is the velocity of the mass, $R_{r}$ is the conversion ratio between the velocity $V_{v}$ and the rotational speed $\omega_{v}, g$ is the gravitational acceleration, $\lambda=1-\frac{\omega_{e m}}{\omega_{v}}$ is the slip velocity, and $\mu(\lambda)=c_{1}\left(1-e^{-c_{2}|\lambda|}-c_{3}|\lambda|\right)$ is a nonlinear function based on Burkhardt model with the coefficients $c_{i}$, respectively [3]. Now, the proposed method corrected the actual dynamics (1) of the test-rig (Figure 6) into the desired dynamics (24a), while the load torque $T_{l}=-R_{r} F_{f}$ was generated by the dynamic model (24b) and (24c), which was also implemented in the dynamometer side. In addition, the motor torque was applied by a nonlinear controller:

$$
\begin{aligned}
\dot{\xi}_{1} & =\xi_{2} \\
T_{B} \dot{\xi}_{2} & =-\xi_{2}+\gamma_{B} \operatorname{sign}\left(\lambda^{*}-\lambda\right) \\
T_{m} & =-\operatorname{sat}\left(\xi_{1}\right)
\end{aligned}
$$

where $\lambda^{*}=0.2$ is the desired slip velocity, $T_{B}$ and $\gamma_{B}$ are the design parameters of the controller, $\operatorname{sign}(\cdot)$ denotes the sign of the input signal $(1$ or -1$)$, and sat $(\cdot)$ represents the saturation function to limit the motor torque within $\left|T_{m}\right|<13 \mathrm{Nm}$.

Remark 4. The dynamics (24) represents the dynamic behavior of a single wheel in a vehicle under the hard braking condition [3], i.e., $F_{f}$ is called adhesion force, which is determined by the slip velocity of the vehicle $\lambda$. The controller (25) is also known as the bang-bang controller in order to prevent skidding of the vehicle. One can easily test the behavior of the closed-loop system (24) and (25) by using the simulation model in MATLAB/Simulink [20]. 
Figure 10 shows the experimental results when the parameters of the desired emulating dynamics (24) were chosen as $m=10 \mathrm{~kg}, J_{e m}=5 \mathrm{~J}, B_{e m}=5 B, g=9.8 \mathrm{~m} / \mathrm{s}^{2}, R_{r}=0.2 c_{1}=0.5, c_{2}=24$, and $c_{3}=0.52$, respectively. The initial conditions of the rotating speeds were given by $\omega_{m}(0)=\omega_{v}(0)=$ $83 \mathrm{rad} / \mathrm{s}$. The simulation results to be compared are denoted by $T_{e m}, \omega_{e v}$ and $\omega_{e m}$ (which correspond to the measurements $T_{m}, \omega_{v}$, and $\omega_{m}$ by emulating (24)), respectively. In the figures, the responses of the torques are slightly different $\left|T_{m}-T_{e m}\right| \leq 2 \mathrm{Nm}$ with the phase difference, while the emulating error $\left|\omega_{m}-\omega_{e m}\right|$ is less than $5 \mathrm{rad} / \mathrm{s}$. The emulating performance degraded in this experiment, because the motor torque was significantly varied by using the sign function (25).
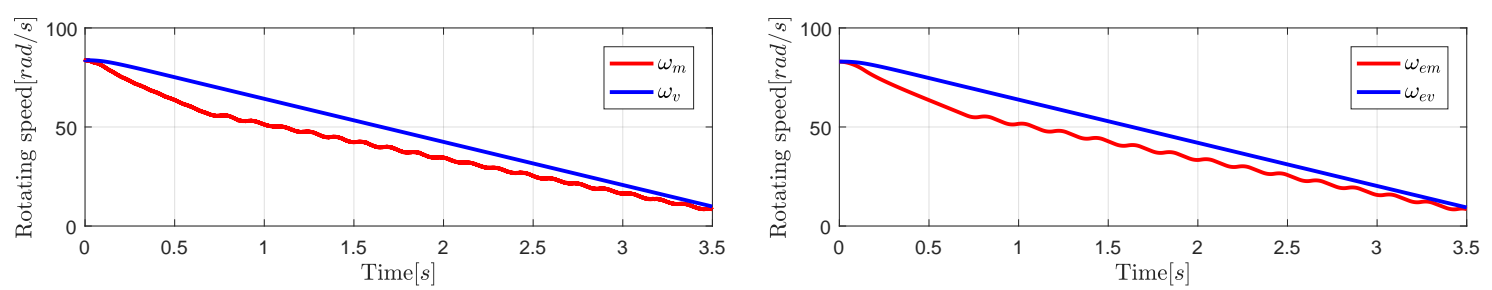

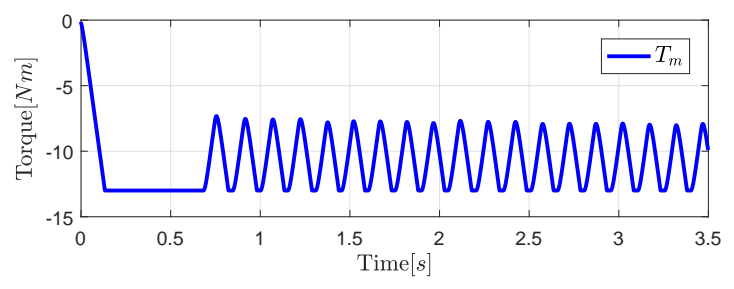

(a)

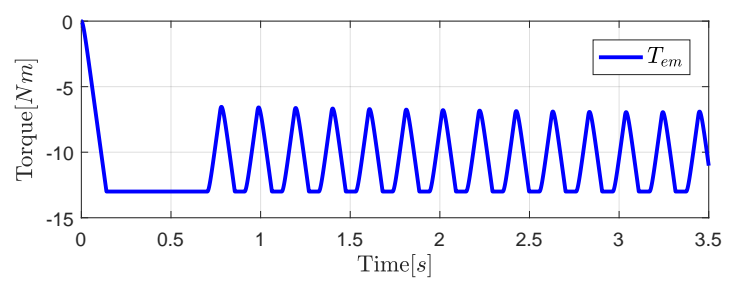

(b)

Figure 10. The responses of the motor torque $T_{m}$ with the nonlinear controller (25): $T_{B}=0.01$ and $\gamma_{B}=100$. (a) The experimental results. (b) The simulation results.

\section{Conclusions}

The property of nominal performance recovery of the disturbance-observer has been exploited to derive a robust emulation scheme. The dynamics to be emulated was considered as the nominal model of the disturbance-observer, and the disturbance-observer endowed the proposed emulation scheme with robustness against plant uncertainties. The range of emulation parameters was wider than previous results, and a rigorous stability analysis was presented considering the uncertainties explicitly, which has not been considered in previous works. The performance of the proposed emulation scheme was verified through experiments, and the effect of different values of tuning parameter has been investigated. It was demonstrated that up to 20-times of inertia emulation with relatively small emulation error (speed error less than 6\%) was achieved, and that various loads such as friction could be emulated. It was observed that as the bandwidth of the filter increased, the emulation accuracy improved, while the control signal became less smooth. Further investigation on this relation will be an interesting future research topic.

Author Contributions: K.L., J.L., J.B. and Y.I.L. contributed to the design and implementation of the research, to the analysis of the results and to the writing of the manuscript.

Funding: This work (Grant No. C0541054) was supported by the Business for Cooperative R \& D between Industry, Academy, and Research Institute-funded Korea Small and Medium Business Administration in 2017 and the Korea Institute of Energy Technology Evaluation and Planning (KETEP) and the Ministry of Trade, Industry \& Energy (MOTIE) of the Republic of Korea (No. 20153010024470).

Conflicts of Interest: The authors declare no conflict of interest. 


\section{References}

1. Leonhard W. Control of Electrical Drives; Springer Science \& Business Media: Berlin, Germany, 2012.

2. Todd, R.; Forsyth, A.J. HIL emulation of all-electric UAV power systems. In Proceedings of the IEEE Energy Conversion Congress and Exposition, San Jose, CA, USA, 2009; pp. 411-416.

3. Li, L.; Wang, F.-Y.; Zhou, Q. Integrated longitudinal and lateral tire/road friction modeling and monitoring for vehicle motion control. IEEE Transa. Intell. Transp. Syst. 2006, 7, 1524-9050. [CrossRef]

4. Song, S.H.; Jeong, B.C.; Lee, H.I.; Kim, J.J.; Oh, J.H.; Venkataramanan, G. Emulation of output characteristics of rotor blades using a hardware-in-loop wind turbine simulator. In Proceedings of the Twentieth Annual IEEE Applied Power Electronics Conference and Exposition, Austin, TX, USA, 6-10 March 2005; pp. 1791-1796.

5. Gan, C.; Todd, R.; Apsley, J. Time-delay effects in a HIL aircraft power system emulator. In Proceedings of the IEEE Energy Conversion Congress and Exposition, Denver, CO, USA, 15-19 September 2013; pp. $20-26$.

6. Collins, E.R.; Huang, Y. A programmable dynamometer for testing rotating machinery using a three-phase induction machine. IEEE Trans. Energy Convers. 1994, 9, 521-527. [CrossRef]

7. Sandholdt, P.; Ritchie, E.; Pedersen, J.K.; Betz, R.E. A dynamometer performing dynamical emulation of loads with nonlinear friction. IEEE Int. Symp. Ind. Electron. 1996, 2, 873-878.

8. Akpolat, Z.H.; Asher, G.M.; Clare, J.C.; Dynamic emulation of mechanical loads using a vector-controlled induction motor-generator set. IEEE Trans. Ind. Electron. 1999, 46, 370-379. [CrossRef]

9. Arellano-Padilla, J.; Asher, G.M.; Sumner, M. Control of an AC Dynamometer for Dynamic Emulation of Mechanical Loads With Stiff and Flexible Shafts. IEEE Trans. Ind. Electron. 2006, 53, 1250-1260. [CrossRef]

10. Kyslan, K.; Ďurovskỳ, F. Dynamic emulation of mechanical loads-An approach based on industrial drives' features. Automatika-J. Control Meas. Electron. Comput. Commun. 2013, 54, 356-363. [CrossRef]

11. Back, J.; Lee, K.; Choy, I.; Park, J.W.; Kim, H.E. Emulation of dynamic mechanical load for dynamometer using disturbance-observer based robust controller. In Proceedings of the International Conference on Control, Automation and Systems, Singapore, 30-31 July 2011; pp. 1037-1040.

12. Umeno, T.; Hori, Y. Robust speed control of DC servomotors using modern two degrees-of-freedom controller design. IEEE Trans. Ind. Electron. 1991, 38, 363-368. [CrossRef]

13. Ryoo, J.R.; Doh, T.Y.; Chung, M.J. Robust disturbance-observer for the track-following control system of an optical disk drive. Control Eng. Pract. 2004, 2, 577-585. [CrossRef]

14. Chen, W.H.; Yang, J.; Guo, L.; Li, S. Disturbance-Observer-Based Control and Related Methods-An Overview. IEEE Trans. Ind. Electro. 2016, 63, 1083-1095. [CrossRef]

15. Back, J.; Shim, H. Adding robustness to nominal output-feedback controllers for uncertain nonlinear systems: A nonlinear version of disturbance-observer. Automatica 2008, 44, 2528-2537. [CrossRef]

16. Shim, H.; Park, G.; Joo, Y.; Back, J.; Jo, N.H. Yet another tutorial of disturbance-observer: robust stabilization and recovery of nominal performance. Control Theory Technol. 2006, 4, 237-249. [CrossRef]

17. Khalil, H.K. Nonlinear Systems, 3rd ed.; Prentice-Hall: Upper Saddle River, NJ, USA, 2002.

18. Sul, S.K. Control of Electric Machine Drive Systems; John Wiley \& Sons: Hoboken, NJ, USA, 2011.

19. Akpolat, Z.H.; Asher, G.M.; Clare, J.C. Experimental dynamometer emulation of nonlinear mechanical loads. IEEE Trans. Ind. Appl. 1999, 35, 1367-1373. [CrossRef]

20. Modeling an Anti-Lock Braking System, Mathworks. Available online: http://mathworks.com/help/ simulink/examples/modeling-an-anti-lock-braking-system.html (accessed on 9 June 2019).

(C) 2019 by the authors. Licensee MDPI, Basel, Switzerland. This article is an open access article distributed under the terms and conditions of the Creative Commons Attribution (CC BY) license (http://creativecommons.org/licenses/by/4.0/). 